The account given is by far the most comprehensive to date. In Chapter III he considers rectangular bands $(x y x=x$ for all $x, y)$ and what he calls matrix decompositions of a semigroup (such a decomposition is induced by a homomorphism onto a rectangular band). Chapter IV is devoted to normal band decompositions, a normal band $(x y z y=x z y x)$ being, in the variety of bands, the join of a semilattice $(x y=y x)$ and a rectangular band. In Chapter V the main object of study is the lattice of subsemigroups of a semigroup and the obvious problems of when this lattice is modular, distributive, complemented, etc. Nowadays, with Mathematical Reviews coping with more than 2,500 papers per month, it is essential that top notch mathematicians such as Petrich should devote a lot of their time to producing survey research monographs. But, please, in the galaxy of publications let each of these not have the appearance of a white dwarf.

T. S. BLYTH

\title{
Kolman, B., Introductory Linear Algebra with Applications (Macmillan, 1976), xvi+ 426 pp.
}

This book is intended as an introduction to linear algebra, with applications (in the social sciences), and with some emphasis on numerical linear algebra. To construct such a book is a challenging task and the author has had some success. I am afraid, however, that there are many details about which I am not happy, and which, taken together, tend to diminish the usefulness of the book. Since the text falls fairly clearly into three parts I shall discuss these separately.

First, the theoretical linear algebra part is constrained to finite-dimensional vector spaces over the real field (complex numbers are strictly forbidden, even as eigenvalues of real unsymmetric matrices). An axiomatic approach is used, but several theorems are given without proof (especially concerning matrix rank). No attempt is made to draw a distinction between a vector space (only $\boldsymbol{R}^{n}$ is considered in any detail) and its coordinate space with respect to a basis. Indeed these concepts are identified, and vectors are notated as $n \times 1$ matrices. This use of the same notation for matrices and vectors (capital Clarendon type) makes some expressions unclear. Some abstract theory is presented, but is rather unsatisfactory; the definition of vector space using general operations requires bracketing in places, because of the finite-dimensional assumption linear transformations appear little different from matrices (and are often specified using matrices), and no definition of composition of these is given. It is a pity that the author omitted the unifying result that the general solution of a linear equation is a particular solution plus the general solution of the associated homogeneous equation. Perhaps some of these points could have been improved at the cost of much of the material on determinants, which I found to be excessive in detail, and to come rather early in the text for such a relatively unimportant topic.

Second, the applications examined are linear programming, geometry, graph theory, game theory, least squares, and economic models. None of these involves any calculus. On the whole these are successful, especially the economic models which bring out well the unifying nature of linear algebra. It is a pity that the linear programming material stops short of duality, especially since all the matrix tools are available. The matrix games section is rather spoiled by three errors in the examples and an unclear definition of "constant-sum games", which may well confuse inexperienced readers.

Third, the numerical linear algebra part covers Gaussian elimination with partial pivoting, iterative solution of linear equations, the power method, and Jacobi's eigenvalue method. It is unfortunate that the recommendation of Gauss-Jordan elimination given in Chapter 1 must stand, without comment, until Chapter 8 , especially since the more efficient method can be achieved by using the row operations in a different order, or even by fewer operations. (This latter method is achieved at the cost of uniqueness of the echelon form, but this is seldom useful.) Finally, the text contains the fallacy that it is computationally efficient to find the inverse matrix when several sets of linear equations with different right-hand sides are to be solved.

The text is very clearly printed, with helpful diagrams and tables. There are copious worked examples at all levels of difficulty, with many exercises, solutions being given to half of them. I observed about a dozen misprints, mostly trivial. In addition to the errors already mentioned, the definition of congruence is later contradicted by use of a non-orthogonal transformation, and some of the discussion about degeneracy in linear programming is wrong. 
In view of my reservations I cannot recommend this book for use with specialist mathematics students. Indeed it is not clear that the amount of material presented is suitable for them. But I do recommend it for other disciplines, especially for students of economics and business studies, since the applications are so suitable.

D. W. ARTHUR

Cameron, P. J., Combinatorial Surveys (Proceedings of the Sixth British Combinatorial Conference, Academic Press, 1977.)

This book contains seven of the nine principal lectures given at the 6th British Combinatorial Conference, held at Royal Holloway College, London, during July 1977. The book was in fact on sale at the conference-an excellent idea in my opinion. A wide range of combinatorial topics is covered, with block designs, graphs and projective spaces as the main themes.

The first chapter, by F. Buekenhout ("What is a subspace?") is concerned with making a definition of a subspace of an incidence structure, which obviously must be consistent with existing notions of subspace for certain known incidence structures. The ideas are very general and the author himself admits that little progress is possible without considerable restrictions on the incidence structure.

Chapter 2 (P. J. Cameron, "Extensions of designs; variations on a theme") and Chapter 4 (D. K. Ray-Chaudhuri, "Combinatorial characterization theorems for geometric incidence structures") are good survey articles of the areas mentioned in the titles, presenting many of the known results, giving most of the necessary definitions and indicating some of the proofs. Both articles are relatively easy to read and give the reader a certain amount of feeling for the subject. On the other hand, Chapter 3, (L. Lovász, "Flats in matroids and geometric graphs") is extremely difficult to understand, mainly because none of the basic definitions are given. The author is concerned with covering problems in graphs, which he looks at from the point of view of flats in matroids.

The longest chapter (which incidentally gave rise to the shortest lecture) is by N. J. A. Sloane ("Binary codes, lattices and sphere packings"), in which he investigates connections between error-correcting codes and sphere packings, especially lattice packings. The article becomes very technical in places, but fortunately there are plenty of examples. Several open (and presumably difficult) problems are posed and there is an extensive bibliography.

Perhaps the best article is Chapter 6 (A. T. White, "Graphs of groups on surfaces") in which the author extends some of the ideas in his book of (roughly) the same title, using the recently developed theory of voltage graphs. At the end of the chapter there is a very interesting section on an application of imbeddings of graphs to problems in campanology.

The final chapter by D. R. Woodall ("Zeros of chromatic polynomials") is essentially selfcontained and would be an excellent starting point for somebody wishing to study chromatic polynomials.

Overall, I feel that the book has achieved a nice balance; on the one hand, containing a collection of good expository articles on a wide variety of combinatorial topics, whilst on the other, showing clearly some of the overlap between the various subjects. Finally, the book is well presented, with few typographical errors-a reflection, I think, on the editor, Peter Cameron.

MICHAEL J. GANLEY

PitTie, H. V., Characteristic classes of foliations (Research Notes in Mathematics No. 10, Pitman), $107 \mathrm{pp}$.

In this book, the author attempts a rapid treatment of the theory of primary and secondary characteristic classes of foliations and their relationship to recent work of Gelfand and Fuks on the cohomology of formal vector fields. Much emphasis is put on examples which demonstrate that the characteristic classes described may be non-zero.

I found it hard to judge at what audience the book is aimed. In view of the condensed nature of the arguments, it could hardly serve as a satisfactory introduction to the subject. Nor is it likely to be of great use to the expert in the field (although the reviewer does not count himself among 